\title{
Response to letter to the Editor: The therapeutic strategy of drug re-positioning to induce autophagic cell death in brain malignancy
}

\author{
S. I. Omoruyi ${ }^{1,2} \cdot$ A. Jardine ${ }^{3} \cdot$ S. Prince ${ }^{2}$
}

Accepted: 4 November 2020 / Published online: 13 November 2020

(c) Springer Science+Business Media, LLC, part of Springer Nature 2020

\section{Dear Editor,}

We thank Dr Yoshida for his valuable comments and interest in our study which describes the anti-cancer activity of DS00329, a novel derivative of the anti-psychotic drug phenothiazine, in glioblastoma multiforme (GBM) [1]. We appreciate the information provided by Dr Yoshida that illustrates that the activation of autophagy is an important mechanism by which neurochemical compounds and dopamine receptor D4 antagonists inhibit GBM proliferation [2]. Furthermore, we agree with him that levels of p62/SQSTM1 is an important indicator of the autophagic state of cells and that, in addition to our results showing an increase in LC3-11, it would be important to determine the impact of DS00329 on p62/SQSTM1 levels in glioblastoma cells. Indeed, increased LC3-II levels can be associated with either enhanced autophagosome synthesis or reduced autophagosome turnover. Thus, to differentiate between these two processes, it is important to determine the levels of p62/ SQSTM1 because it is a receptor of polyubiquinated proteins and upon autophagy induction, p62/SQSTM1 is incorporated within an autophagosome and degraded within an autolysosome [3]. The degradation of p62/SQSTM1 is thus an indication of autophagic flux. Since the publication of our manuscript, we have confirmed that DS00329 treatment results in decreased levels of p62/SQSTM1 and thus autophagic flux in the U87 and U251 glioblastoma cells.

S. Prince

sharon.prince@uct.ac.za

1 University of the Western Cape, Department of Medical Bioscience, Faculty of Natural Sciences, Bellville 7530, Cape Town, South Africa

2 University of Cape Town, Division of Cell Biology, Department of Human Biology, Faculty of Health Sciences, Observatory 7925, Cape Town, South Africa

3 University of Cape Town, Department of Chemistry, Faculty of Science, Rondebosch 7701, Cape Town, South Africa

Furthermore, we agree with Dr Yoshida that the Acridine Orange stain alone may not be enough to differentiate autophagosomes from lysosomes as well as autolysosomes. However, this stain together with results showing a reduction in p62/SQSTM1 levels provide evidence that autophagic cargoes are being transferred to the lysosomes for degradation. Importantly, the antipsychotic phenothiazines, thioridazine and chlorpromazine have also been shown to induce autophagic cell death in glioblastoma [4, 5]. Indeed electron microscopy of chlorpromazine treated cells showed large vacuoles containing cargoes, indicative of autophagic flux [4].

In our manuscript we showed that DS00329 induces DNA damage and apoptotic and autophagic cell death in U87 and U251 glioblastoma cells and we are currently investigating the signalling pathways involved. We thus agree with Dr Yoshida that as part of these investigations we should explore the impact of DS00329 on the c-Jun N-terminal kinase (JNK) and p38 MAP kinase pathways because of their roles in autophagic cell death and the DNA damage pathway respectively. Indeed, our preliminary data show that DS00329 activates the p38 MAP kinase pathway in U87 and U251 glioblastoma cells and the JNK pathway in patient derived xenograft glioblastoma cells. Finally, we realize the heightened interest in this research area, and we are currently preparing a review on the repurposing of antipsychotic phenothiazines for the treatment of cancer and their mechanism(s) of actions and we will certainly include the suggestions made by Dr Yoshida. Indeed, chlorpromazine is one of the first phenothiazines that has been registered for a clinical trial for GBM and we will be tracking its progress. It is worth noting that the repurposing of chlorpromazine for Covid-19 treatment is also under investigation. 


\section{References}

1. Omoruyi S, Ekpo O, Semenya D, Jardine A, Prince S (2020) Exploitation of a novel phenothiazine derivative for its anti-cancer activities in malignant glioblastoma. Apoptosis 25:261-274

2. Yoshida GJ (2020) The therapeutic strategy of drug re-positioning to induce autophagic cell death in brain malignancy. Apoptosis 25:457-458. https://doi.org/10.1007/s10495-020-01617-1

3. Pankiv S, Clausen TH, Lamark T, Brech A, Bruun J-A, Outzen H, Øvervatn A, Bjørkøy G, Johansen T (2007) p62/SQSTM1 binds directly to Atg8/LC3 to facilitate degradation of ubiquitinated protein aggregates by autophagy. J Biol Chem 282(33):24131-24145

4. Shin SY, Lee KS, Choi Y-K, Lim HJ, Lee HG, Lim Y, Lee YH (2013) The antipsychotic agent chlorpromazine induces autophagic cell death by inhibiting the Akt/mTOR pathway in human U-87MG glioma cells. Carcinogenesis 34(9):2080-2089. https://doi.org/10.1093/carcin/bgt169

5. Cheng H-W, Liang Y-H, Kuo Y-L, Chuu C-P, Lin C-Y, Lee M-H, Wu AT, Yeh C-T, Chen EI, Whang-Peng J (2015) Identification of thioridazine, an antipsychotic drug, as an antiglioblastoma and anticancer stem cell agent using public gene expression data. Cell Death Dis 6(5):e1753

Publisher's Note Springer Nature remains neutral with regard to jurisdictional claims in published maps and institutional affiliations. 